\title{
Fluctuations in brain temperature induced by lypopolysaccharides Central and peripheral contributions
}

\author{
Jeremy S. Tang and Eugene A. Kiyatkin* \\ Behavioral Neuroscience Branch; National Institute on Drug Abuse; Intramural Research Program; National Institutes of Health; DHHS; Baltimore, MD USA
}

Key words: brain, muscle and skin temperatures, hypothalamus, metabolic activation, vasoconstriction, vasodilatation, rats

Abbreviations: ANOVA, analysis of variance; iv, intravenous; LPS, lypopolysaccharides; MPAH, medial preoptic-anterior hypothalamus

In this study, we examined changes in central (anterior-preoptic hypothalamus) and peripheral (temporal muscle and facial skin) temperatures in freely moving rats following intravenous administration of bacterial lipopolysaccharides (LPS) at low doses $(1$ and $10 \mu \mathrm{g} / \mathrm{kg})$ at thermoneutral conditions $\left(28^{\circ} \mathrm{C}\right)$. Recordings were made with high temporal resolution (5-s bin) and the effects of LPS were compared with those induced by a tail-pinch, a standard arousing somato-sensory stimulus. At each dose, LPS moderately elevated brain, muscle and skin temperatures. In contrast to rapid, monophasic and relatively short hyperthermic responses induced by a tail-pinch, LPS-induced increases in brain and muscle temperatures occurred with $\sim 40$ min onset latencies, showed three not clearly defined phases, were slightly larger with the $10 \mu \mathrm{m} / \mathrm{kg}$ dose and maintained for the entire 4-hour post-injection recording duration. Based on dynamics of brain-muscle and skin-muscle temperature differentials, it appears that the hyperthermic response induced by LPS at the lowest dose originates from enhanced peripheral heat production, with no evidence of brain metabolic activation and skin vasoconstriction. While peripheral heat production also appears to determine the first phase of brain and body temperature elevation with LPS at $10 \mu \mathrm{g} / \mathrm{kg}$, a further prolonged increase in brain-muscle differentials (onset at 100 $\mathrm{min}$ ) suggests metabolic brain activation as a factor contributing to brain and body hyperthermia. At this dose, skin temperature increase was weaker than in temporal muscle, suggesting vasoconstriction as another contributor to brain/ body hyperthermia. Therefore, although both LPS at low doses and salient sensory stimuli moderately increase brain and body temperatures, these hyperthermic responses have important qualitative differences, reflecting unique underlying mechanisms.

\section{Introduction}

Brain temperature is an important physiological parameter that affects numerous neural functions. Despite erroneous beliefs in its stability and tight regulation, brain temperature fluctuates within relatively large limits $\left(3-4^{\circ} \mathrm{C}\right)$ in the normal physiological and behavioral continuum. ${ }^{1,2}$ In rats, temperatures in deep brain structures fall to its nadir $\left(-35^{\circ} \mathrm{C}\right)$ during slow-wave sleep and increase up to $38-39^{\circ} \mathrm{C}$ following salient environmental stimulation and during different types of naturally motivated behaviors. ${ }^{3-5}$ Despite minor structural differences evident with high-resolution recordings, these temperature fluctuations are generally correlative in various brain structures and associated with similar changes in body core temperatures. Therefore, brain and body temperature homeostasis could be altered under physiologically relevant conditions via a purely neural mechanism, involving stimulation of somato-sensory afferents, rapid neurotransmission and subsequent metabolic brain activation.

These physiological temperature fluctuations that appear to be a part of normal organism's adaptive activity stand apart from fever or "controlled hyperthermia" that is induced by a number of toxins as a part of the organism's immune response during bacterial or viral infection. ${ }^{6,7}$ While manifestations of fever greatly vary across different animal species, depending upon the nature and levels of toxins and an organism's individual responsiveness to them, in rodents fever is usually modeled with systemic administration of lipopolysacharides (LPSs), the best-known exogenous pyrogens. In contrast to rapid, monophasic and relatively shortterm hyperthermic responses induced by salient somato-sensory stimuli, LPS-induced body temperature elevation develops with much longer onset latencies, has several phases, and is continued for at least several hours or more. ${ }^{8,9}$ While an enhanced metabolism-related heat production and diminished heat dissipation due 
to peripheral vasoconstriction are usually viewed as the primary factors responsible for LPS-induced body temperature elevation, the relationship between these two factors and the role of the brain in mediating the temperature responses remain unclear. It is generally believed that the hypothalamus is the primary site that triggers LPS-induced hyperthermia (reviewed in ref. 7 and 10), but LPSs minimally cross the blood-brain barrier. ${ }^{11,12}$ Instead, they induce the release of multiple endogenous pyrogens (i.e., interleukin 1 and 6 , tumor necrosis factor- $\alpha$, prostaglandins), which could act both in the brain and periphery to increase metabolism and heat production. ${ }^{13-15}$ It is unclear, however, whether this effect is triggered centrally (i.e., via brain metabolic activation and subsequent involvement of sympathetic mechanisms) or results from the direct action of endogenous pyrogens on peripheral heat-producing organs (i.e., liver, muscle, adipose tissue). While data on LPS-induced changes in brain metabolism are limited and inconclusive, LPSs enhance bodily heat production and energy expenditure by increasing expression of uncoupling protein- $2 \mathrm{mRNA}$ in peripheral tissues (liver, muscles, white adipose tissue). ${ }^{16,17}$ Numerous substances released following LPS administration (i.e., interleukins, prostaglandins, NO, bradykinin) could also act both centrally and directly on blood vessels to induce peripheral vasoconstriction, another contributor to LPSinduced temperature elevation.

Although there are numerous studies that describe body temperature effects of LPSs in various animal species and at different doses, their effects on brain temperature and their relationships with peripheral temperatures remain unknown. To clarify this issue, we examined changes in hypothalamic (medial preopticanterior hypothalamus or MPAH) and peripheral (temporal muscle and facial skin) temperatures induced by LPS in freely moving rats. We used representative Escherichia coli LPSs that were delivered intravenously (iv) at very low doses (1 and $10 \mu \mathrm{g} /$ $\mathrm{kg}$ ) that are known to increase body temperatures in rats. LPS and saline were administered to animals intensively habituated to the testing environment via a chronically implanted jugular catheter and from a distant location, thus providing quiet resting conditions for temperature baselines and eliminating the arousing effect of the injection procedure. ${ }^{9}$ Our measurements were conducted at $27-29^{\circ} \mathrm{C}$, which correspond to thermoneutral conditions, i.e., when heat production and heat loss are balanced and metabolism is at the lowest rates. ${ }^{18,19}$ LPS-induced temperature changes were compared with those induced by a tail-pinch, a typical arousing stimulus that induces brain and body hyperthermic responses coupled with acute peripheral vasoconstriction via a purely neural mechanism. ${ }^{20}$ To capture rapid fluctuations, temperatures were recorded with high temporal resolution (5-s bin) and supplemented with a simultaneous recording of locomotor activity.

While our primary goal was to evaluate the pattern and timecourse of LPS-induced changes in hypothalamic temperature, two other head locations were important for assessing the contributions of arterial blood inflow and the vessel state (vasoconstriction/vasodilatation) to brain temperature fluctuations. Temporal muscle is a non-motor (non-thermogenic) muscle and its temperature depends primarily upon the temperature of arterial blood inflow. Since temporal muscle and the brain receive their arterial blood inflow from the same carotid artery, brain-muscle temperature differentials (i.e., difference in their relative temperatures) can help determine whether brain temperature changes come from central (i.e., intra-brain heat production) or peripheral source. As shown in our previous studies, brain temperature increases induced by salient environmental stimuli and psychomotor stimulants are consistently more rapid and stronger than those in temporal muscle (reviewed in ref. 20 and 21), suggesting metabolic brain activation as a primary triggering factor of brain hyperthermia. In contrast, the relationships between brain and muscle temperatures are opposite during pentobarbital-induced anesthesia, with much stronger decreases in brain structures than in the muscle or body core, pointing at central metabolic inhibition as a leading factor of brain hypothermia. ${ }^{22}$ Skin temperature depends primarily upon the vessel state and its absolute change indicates the extent of heat loss to the external environment. Since skin temperature is also affected by slower temperature influences from the arterial blood supply, skin-muscle temperature differentials provide more accurate evaluation of vasoconstriction/vasodilatation by excluding this influence. Using this approach, we previously showed that despite a decrease in absolute skin temperature during pentobarbital anesthesia, this change is much weaker than that in temporal muscle, suggesting tonic vasodilatation as an important contributor to drug-induced hypothermia. ${ }^{22}$ Skin-muscle differentials could be especially important for evaluating a vascular change following such slow and prolonged temperature responses as those induced by LPS.

\section{Results}

The present data were obtained from 8 rats that were recorded during multiple sessions conducted within a two-week time interval. Each rat received three LPS injections (one at 1 and two at $10 \mu \mathrm{g} / \mathrm{kg}$ ), 2-3 injections of saline and two tail-pinch presentations.

Basal temperatures and locomotion: effects of saline and recording time: temperature and motor responses to tail-pinch. Consistent with our previous work, rats intensively habituated to the recording environment were generally inactive and had basal hypothalamic temperatures varying between $36-37^{\circ} \mathrm{C}$. Temperatures in temporal muscle were consistently lower than in the MPAH both in each individual animal and as group means $\left(36.02 \pm 0.19^{\circ} \mathrm{C}\right.$ vs. $\left.36.81 \pm 0.19^{\circ} \mathrm{C} ; \mathrm{p}<0.01\right)$. Facial skin had the lowest temperature $\left(35.20 \pm 0.12^{\circ} \mathrm{C}\right)$, significantly differing from that in both the muscle and brain.

As shown in Figure 1 (left part), slow saline administration $(0.3 \mathrm{ml}$ within $20 \mathrm{~s})$ did not induce any significant changes in temperature and locomotion. However, brain and muscle temperatures slowly and gradually decreased within a session, while skin temperature was either stable or slightly increased (A1 and B2). Importantly, brain-muscle differentials remained highly stable within the entire analysis interval, while skin-muscle differential slightly increased within a two-hour observation period (C1). Despite much higher variability (see standard errors in Fig. 1C), the effect was significant. Although saline injection resulted 


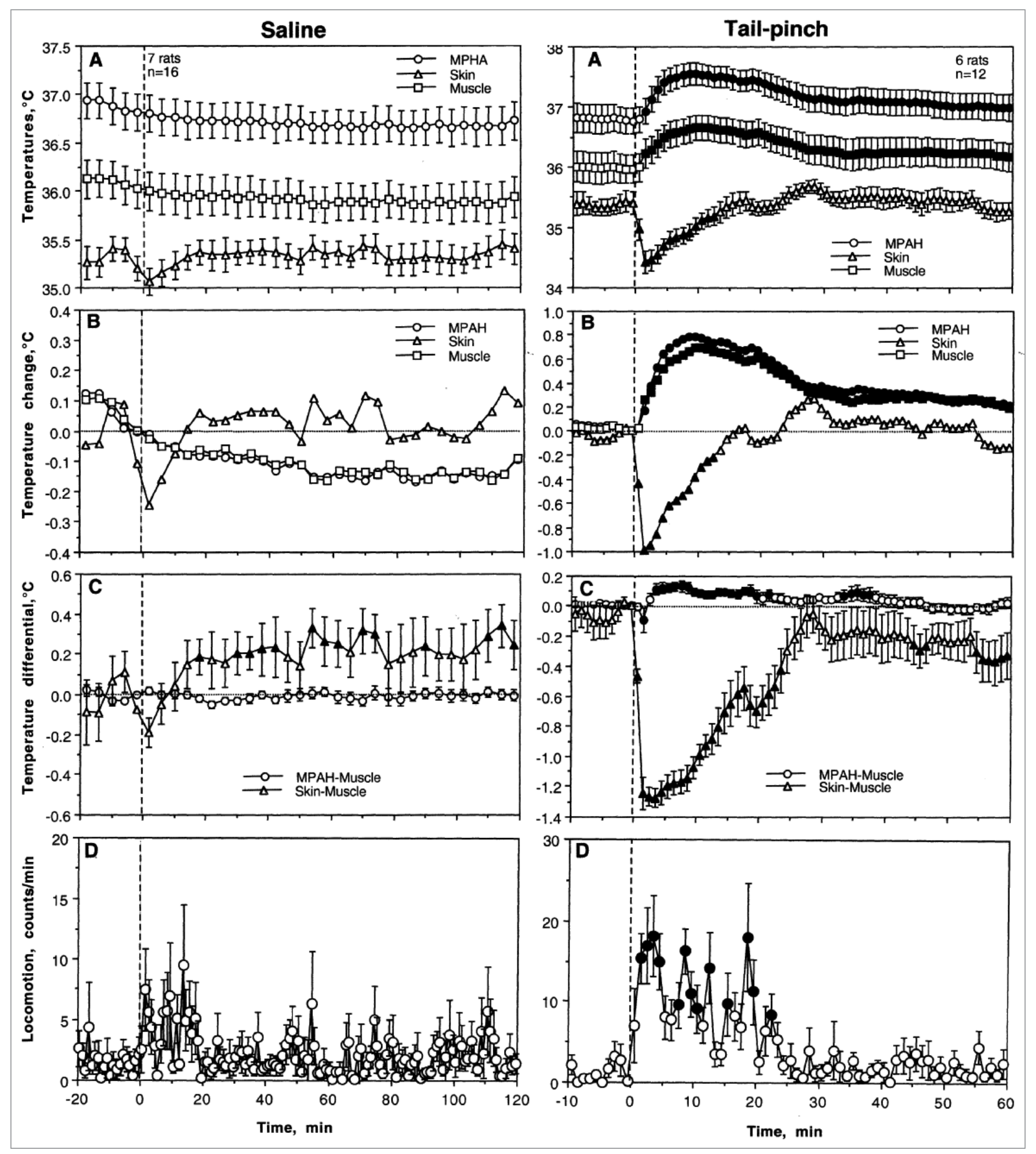

in a weak increase in locomotion within -20 min post-injection, this effect did not reach significance (D).

Although saline was administered via a catheter tubing from a distant location in order to make these injections undetectable by the rat, we could not exclude that a weak locomotor response and transient decrease in skin temperature seen immediately before and after saline administration could result from sensory stimulation related to the injection procedure. A weak increase in skin-muscle differential seen during the session could reflect a gradual weakening of skin vasoconstriction following animal habituation to the warm housing environment.

Consistent with our previous work, tail-pinch-induced locomotor activation and robust temperature responses in each recording location (Fig. 1, right part). Brain and muscle temperatures 
Figure 1 (See opposite page). Changes in brain (MPAH), temporal muscle and facial skin temperatures following iv saline administration (left part) and one-min tail-pinch (right part) in freely moving rats maintained at thermoneutral conditions $\left(27-29^{\circ} \mathrm{C}\right)$. The figure shows: (A) absolute temperatures; (B) relative temperatures; (C) MPAH-muscle and skin-muscle temperature differentials; and (D) locomotion. Temperature data for saline are shown with $4 \mathrm{~min}$ averaging and for tail-pinch with 1-min averaging. Locomotor data for both tests are shown as a number of counts per either 4 (saline) or 1-min (tail-pinch) intervals. Statistical analyses were conducted with one-way ANOVA for repeated measures. The effect of time on temperatures for saline injection was not significant $\left(F_{15,495}=0.75,0.63\right.$ and 1.10, for MPAH, muscle and skin, respectively), but highly significant for tail-pinch $\left(F_{12,402}=19.61,14.58\right.$ and 27.51 for MPAH, muscle and skin, respectively; each $\left.p<0.001\right)$. ANOVA values for the effects on brain-muscle differentials: saline- $F_{15,495}=0.42, p=0.99$; tail-pinch $-F_{12,402}=4.13(p<0.001)$ and on skin-muscle differentials: saline $-F_{15.495}=1.59(p<0.03) ;$ tail-pinch- $F_{12,402}=26.05$ $(p<0.001)$. The effect on locomotion was not significant for saline $(p=0.11)$, but highly significant for tail-pinch $(p<0.001)$. Values significantly different from baselines are shown by filled symbols.

moderately increased (peak of $\sim 0.8^{\circ} \mathrm{C}$ at $\sim 10 \mathrm{~min}$ ) and generally paralleled. However, there were minor but highly significant changes in MPAH-muscle temperature differentials: a transient decrease (1-2 $\mathrm{min}$ ) followed by a more prolonged increase (4-20 min). Skin temperature rapidly and strongly decreased during tail-pinch, with a nadir during the first minute following tailpinch. Changes in skin-muscle differentials indicate strong and relatively long-term $(-25 \mathrm{~min})$ skin vasoconstriction. Locomotor activation was strong, greatly exceeding the duration of tail-pinch $(-20 \mathrm{~min})$.

Effects of LPSs. As can be seen in Figure 2 (left part), brain, muscle and skin temperatures moderately increased following LPS injection $(1 \mu \mathrm{g} / \mathrm{kg})$. The increase in brain and muscle temperatures $\left(-0.8^{\circ} \mathrm{C}\right)$ was about the same as with a tail-pinch, but occurred with -48 min latencies (which were about the same in both locations) and had three not clearly defined phases (peaks at $-60,-140 \mathrm{~min}$ and in the end of recording). The changes in both temperatures paralleled (A and $\mathrm{B})$ and the MPAH-muscle differential fluctuated around zero for the entire recording interval (C). Increase in skin temperature occurred with a slightly shorter latency $(32 \mathrm{~min})$ and showed a transient rise at the time when brain and muscle temperatures showed significant increases. Skin-muscle differentials showed robust fluctuations both before and after drug administration but transiently (and significantly) increased around the time when brain and muscle showed the first temperature elevation. LPSs at the lowest dose did not affect locomotor activity (D).

The changes induced by LPS at $10 \mu \mathrm{g} / \mathrm{kg}$ dose were similar to those seen at a lower dose, but brain and muscle temperature increases were stronger $\left(-1.0^{\circ} \mathrm{C}\right)$, three phases were clearly evident (peaks at $-60,120-140 \mathrm{~min}$ and at the recording end) and temperature elevation in the skin was weaker than that in the muscle and brain (A and B). Similar to the low dose, LPS at $10 \mu \mathrm{g} / \mathrm{kg}$ had no effect on locomotor activity (D). There were, however, important differences in temperature differentials (C). Although the increases in brain and muscle temperatures occurred with the same latencies ( $44 \mathrm{~min}$ ), which were close to those seen with $1 \mu \mathrm{g} / \mathrm{kg}$ dose, the brain-muscle differential significantly decreased during the first phase of temperature elevation (60-80 $\mathrm{min}$ ) but then significantly increased, correlating with the second phase of temperature elevation $(-80-200 \mathrm{~min})$. Despite a strong increase in absolute skin temperature, skin-muscle differentials phasically decreased during the first phase of temperature elevation and remained tonically at this level for the entire recording interval (C). Because of the 4 hour limit of post-injection recording interval, we were unable to observe the third phase of temperature elevation (onset $-200-210 \mathrm{~min}$ ) in its entirety. However, it appears that this later phase was also related to the rise in brain-muscle differential and a new phase of vasoconstriction $(\mathrm{C})$.

Because of significant differences in MPAH-muscle differentials found with LPS at $10 \mu \mathrm{g} / \mathrm{kg}$ during group analysis, we evaluated dynamics of this parameter in each tested rat (Fig. 3). For this analysis, two injections were averaged in each rat and the resulting data were smoothened for three consecutive data points. As seen in Figure 3, in 6/8 rats, brain-muscle differential increased at $-80-100$ min (arrows) and these increases were consistently preceded by decreases below pre-injection baseline. Despite a later (Rat No. 1, $-190 \mathrm{~min}$ ) or weaker (Rat No. 5) change, a similar pattern was seen in two remaining rats. Interestingly, being calculated from the lowest point, the increases in MPAHmuscle differentials were evident in each rat, varying from 0.2 to $0.4^{\circ} \mathrm{C}$, a range consistently seen with somato-sensory stimuli and psychomotor stimulants. ${ }^{21}$ Similarly, an average increase in this parameter was about $0.3^{\circ} \mathrm{C}$ from the preceding lowest point (a hatched horizontal line in Fig. 2C).

It is known that thermogenic effects of LPSs show tolerance with repeated administration. ${ }^{6}$ Although we had a 48 hour interval after the first LPS injection at the lowest dose and 72-hour intervals between two injections of LPS at $10 \mu \mathrm{g} / \mathrm{kg}$, we compared temperature effects induced by LPS at $10 \mathrm{mg} / \mathrm{kg}$ with the first and second administrations. As shown in Figure 4, temperature dynamics in each recording location was virtually identical with both LPS injections. There were also identical values of basal temperatures in each recording location.

Since our basic analysis of temperature effects of LPS/saline and tail-pinch was conducted with relatively large time bins (3 and $1 \mathrm{~min}$, respectively), it is possible that these stimuli induce rapid, transient changes, which could be revealed with fine temporal resolution. To test for this possibility, we analyzed the immediate effects of tail-pinch, LPS at $10 \mu \mathrm{g} / \mathrm{kg}$ and saline on MPAH and skin temperatures with 5-s time resolution. In contrast to tail-pinch, which induced rapid increase in $\mathrm{MPAH}$ temperature (latency to significant increase $-60 \mathrm{~s}$ ), MPAH temperatures were stable within the first 3 min after both injections. In contrast, skin temperatures showed very rapid decrease during tail-pinch (latency $-20 \mathrm{~s}$ ). Although skin temperatures also slightly decreased after saline and LPS injections and the effects of time for LPS was significant in both cases, there were no significant differences between saline and LPS. This trend to decrease seen in both injection groups possibly reflects uncontrolled sensory influences associated with the injection procedure. 

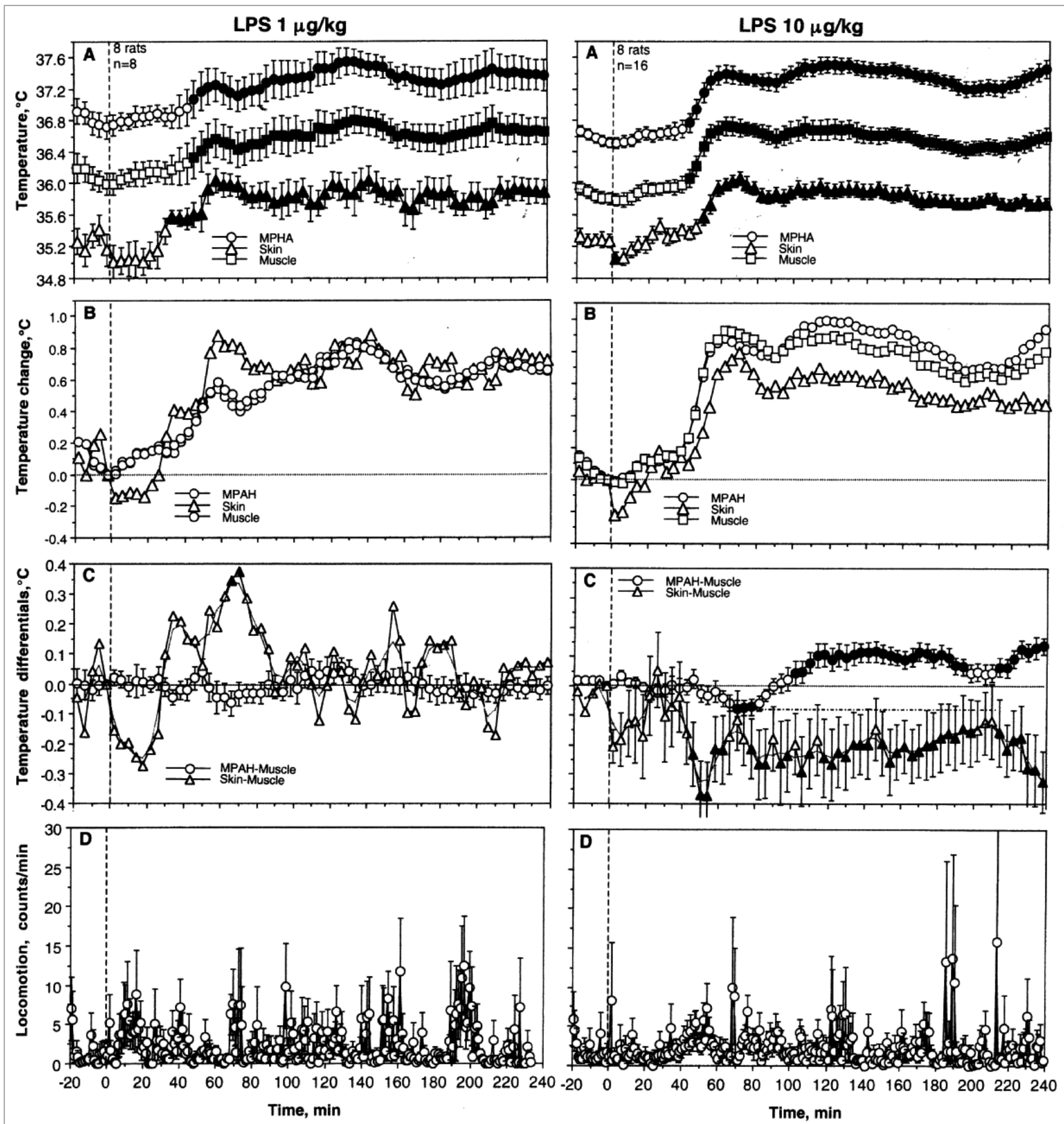

Figure 2. Changes in brain (MPAH), temporal muscle and facial skin temperatures following iv administration of LPS at $1 \mu \mathrm{g} / \mathrm{kg}$ (left part) and $10 \mu \mathrm{g} /$ $\mathrm{kg}$ dose (right part) in freely moving rats maintained at thermoneutral conditions (27-29 $\mathrm{C}$ ). The figure shows: (A) absolute temperatures; (B) relative temperatures; (C) MPAH-muscle and skin-muscle temperature differentials; and (D) locomotion. The effect of time on temperatures was significant for both LPS doses $\left(1 \mu \mathrm{g} / \mathrm{kg}: \mathrm{F}_{7,247}=4.54,4.13\right.$ and 6.99 for MPAH, muscle and skin respectively; $10 \mu \mathrm{g} / \mathrm{kg}: \mathrm{F}_{15,495}=29.12,29.18$ and 19.58 for MPAH, muscle and skin, respectively; each at least $p<0.01)$. The effect of brain-muscle differentials was not significant for $1 \mu \mathrm{g} / \mathrm{kg}\left(\mathrm{F}_{7.247}=1.35, \mathrm{p}=0.11\right)$, but highly significant for $10 \mu \mathrm{g} / \mathrm{kg}\left(F_{15.495}=4.27, \mathrm{p}<0.001\right)$. The effect of skin-muscle differentials was significant for both $1 \mu \mathrm{g} / \mathrm{kg}\left(F_{7.247}=1.86, p<0.01\right)$ and $10 \mu \mathrm{g} / \mathrm{kg}\left(\mathrm{F}_{15,495}=1.69, \mathrm{p}<0.02\right)$. The effect on locomotion was not significant for both doses. Values significantly different from baselines are shown by filled symbols. 


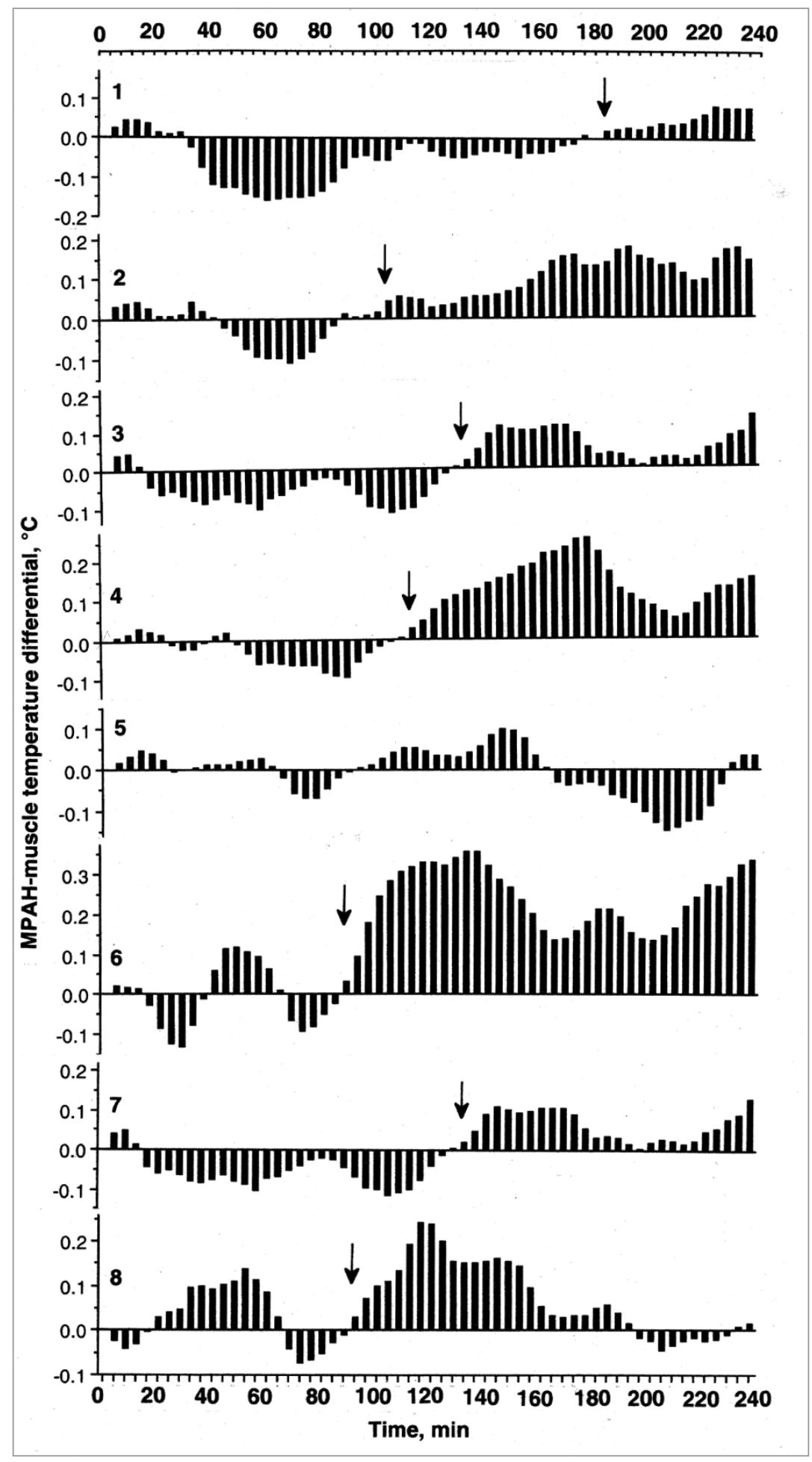

Figure 3. Changes in brain-muscle differentials following LPS administration at $10 \mu \mathrm{g} /$ $\mathrm{kg}$ dose. Each graph shows dynamics of changes in individual rats. Data were averaged for two LPS injections, smoothened (three consecutive values) and shown as a postinjection change. Arrows show time when MPAH-muscle differentials increased above zero line.

\section{Discussion}

This study revealed that iv administration of LPSs at low doses that do not affect spontaneous animal locomotion moderately increase temperatures in the hypothalamus, temporal muscle and facial skin. While hypothalamic and muscle temperatures generally paralleled, skin temperature showed different dynamics. The magnitude of brain and muscle temperature elevation induced by LPSs was comparable to that induced by tailpinch, but their patterns drastically differed. In contrast to relatively short (tens of seconds) latencies seen with tail-pinch, LPS-induced brain and muscle temperature elevation developed with -40 min latencies despite a rapid drug delivery via an iv route. This finding agrees with other observations (reviewed in ref. 6, 8 and 9), supporting the view that not LPSs per se but some other pyrogenic substances released following LPS impact trigger a temperature increase. Both interleukin- $1 \beta$ and prostaglandins induce hyperthermia, which has shorter onset latencies than that induced by LPS. ${ }^{23-25}$ The involvement of multiple endogenous pyrogens as well as a relatively slow clearance of LPS after iv injection could determine a long duration of hyperthermia, which well exceeded a 4 hour postinjection recording interval with both low drug doses used in this study. This feature also differs from relatively short, monophasic hyperthermic responses induced by salient environmental stimuli. In contrast, LPS-induced temperature elevation had at least three phases, especially evident with $10 \mu \mathrm{g} / \mathrm{kg}$ dose. Surprisingly, despite a ten-fold difference in dose, the effects of LPSs were comparable in latencies, amplitudes and durations, but with a $10 \mu \mathrm{g} / \mathrm{kg}$ dose the increases in brain and muscle temperature were slightly stronger $(-1.0 \mathrm{vs}$. $0.7^{\circ} \mathrm{C}$ ), three phases were more clearly defined and changes in skin temperature differed from those seen with a $1 \mu \mathrm{g} / \mathrm{kg}$ dose.

Similar to that seen with tail-pinch and other sensory stimuli, LPS-induced fluctuations in hypothalamic temperature generally paralleled those in temporal muscle, suggesting that LPSs similarly affect brain and body temperature homeostasis. However, there were important differences in dynamics of brain-muscle differentials. Changes in brain-muscle differentials, moreover, also differed for 1 and $10 \mu \mathrm{g}$ LPS doses. While brain and muscle temperatures tightly correlate in quiet resting conditions and brain-muscle differentials remain stable fluctuating around zero (see control in this study), after exposure to somato-sensory stimuli, temperature in the brain increases more rapidly and strongly than in temporal muscle, with a transient 
rise $\left(-0.2-0.3^{\circ} \mathrm{C}\right)$ of brain-muscle differentials. These differentials were surprisingly stable following LPS administration at the lowest dose (see Fig. 2C) despite increases in brain and muscle temperatures. In contrast, LPS at a $10 \mu \mathrm{g} / \mathrm{kg}$ dose produced a biphasic change in brain-muscle differentials, with the initial weak decrease $(-40-60 \mathrm{~min}$, at the time when both temperatures increased) followed by a stronger and more prolonged increase (80-200 min). Although these changes were relatively small in amplitude $\left(0.2-0.3^{\circ} \mathrm{C}\right)$, they were highly significant and similar in range to those seen following somato-sensory stimulation. While it is quite difficult to prove it by independent measure, such dynamics of brain-muscle differentials may suggest that the first phase of temperature elevation induced by LPSs is mediated preferentially via an increased peripheral heat production. If the peripheral heat production and body core temperature increase, arterial blood temperature also rises, thus promoting intra-brain heat accumulation and increasing brain temperatures. Although there is a long-standing discussion regarding the role of cerebral blood flow in regulating brain temperature (reviewed in ref. 21 and 26), heat per se cannot be delivered to the brain from the periphery because of a constant positive temperature gradient between brain tissue and arterial blood arriving to the brain..$^{1-3,27-29}$ This temperature gradient is consistently positive in different animal species, including humans and under different experimental conditions, including deep anesthesia when brain and body temperatures fall $3-5^{\circ} \mathrm{C}$ below their normal, quiet resting baseline. ${ }^{2,30}$ Although brain circulation is the primary means of heat dissipation from the brain to the body and then to the external environment, the rise in arterial blood temperature due to increased peripheral heat production could influence brain temperature homeostasis by preventing proper dissipation of braingenerated metabolic heat.

While the decrease in MPAH-muscle differentials seen at 60-80 min after LPS injection at $10 \mu \mathrm{g} / \mathrm{kg}$ dose is indicative of a peripheral source of heat production (or of its larger contribution than a central source), the subsequent inversion of these differentials (with stronger temperature increases in the hypothalamus than in muscle) could suggest that the second phase of brain temperature elevation is triggered via metabolic brain activation and subsequent involvement of central mechanisms that increase body metabolism. Careful comparison of temperature dynamics (see Fig. 2B and C) reveals that the second phase of temperature increase and an increase in MPAH-muscle differential are present, albeit at a minimal extent, with the threshold LPS dose. A relative "silence" of the hypothalamus following LPS injections at threshold doses and during the initial stage of hyperthermia at higher (but still low) doses could be viewed as surprising since this brain structure is usually viewed as being critical for triggering a hyperthermic response. However, this finding is supported
LPS $10 \mu \mathrm{g} / \mathrm{kg}$ : First vs. second injection

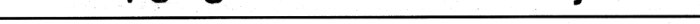

Figure 4. Differences in temperature responses induced by LPS at $10 \mu \mathrm{g} / \mathrm{kg}$ following the first and second injections separated by a 72 hour interval. The graph shows changes in absolute temperature in the MPAH, facial skin and temporal muscle before and after iv LPS administration (0 $\mathrm{min})$. Standard errors are omitted for clarity.

by recent data suggesting early release of prostaglandins in macrophages of the lungs and liver but not in brain tissue. ${ }^{31}$ Both prostaglandins as well as interleukins and uncoupling proteins that are released in the periphery increase heat-producing activity in peripheral organs. ${ }^{32-35}$ In addition, these substances affect multiple afferent pathways to the brain, thus transmitting a signal from the periphery and inducing metabolic brain activation. A relative prevalence of heat-producing activity of MPAH during the second, more prolonged phase of hyperthermia agrees well with profound rise in Fos-like immunoreactivity found in this area at the second hour following LPS administration at comparable doses $\left(5 \mu \mathrm{g} / \mathrm{kg}\right.$, iv). ${ }^{36}$ This measure of biochemical or metabolic activation was profoundly enhanced with larger LPS doses. Fos expression was also increased by LPSs at the same time scale in numerous cortical and subcortical structures that project to the hypothalamus and in specific populations of hypothalamic and brainstem neurons that project to the spinal cord. ${ }^{37,38}$ These latter data point at centrally mediated sympathoexcitation that also leads in peripheral heat production, thus contributing to overall temperature elevation. A relative "silence" of the hypothalamus at the initial stage of LPS-induced temperature elevation does not mean that the hypothalamus and other brain structures are not activated at early stages following LPS impact. Using high-speed amperometry, it was shown that ATP is clearly increased within the anterior hypothalamus at about $18 \mathrm{~min}$ and peaked at -45 min after iv LPS administration in awake rabbits. ${ }^{39}$

In contrast to the rapid, strong and transient decrease in facial skin temperature induced by tail-pinch (see Fig. 1), LPSs at both doses increased these temperatures, suggesting enhanced heat loss from these skin surfaces. However, changes in skin-muscle differentials, which allow for a more accurate evaluation of a vascular effect (vasoconstriction vs. vasodilatation) by excluding 


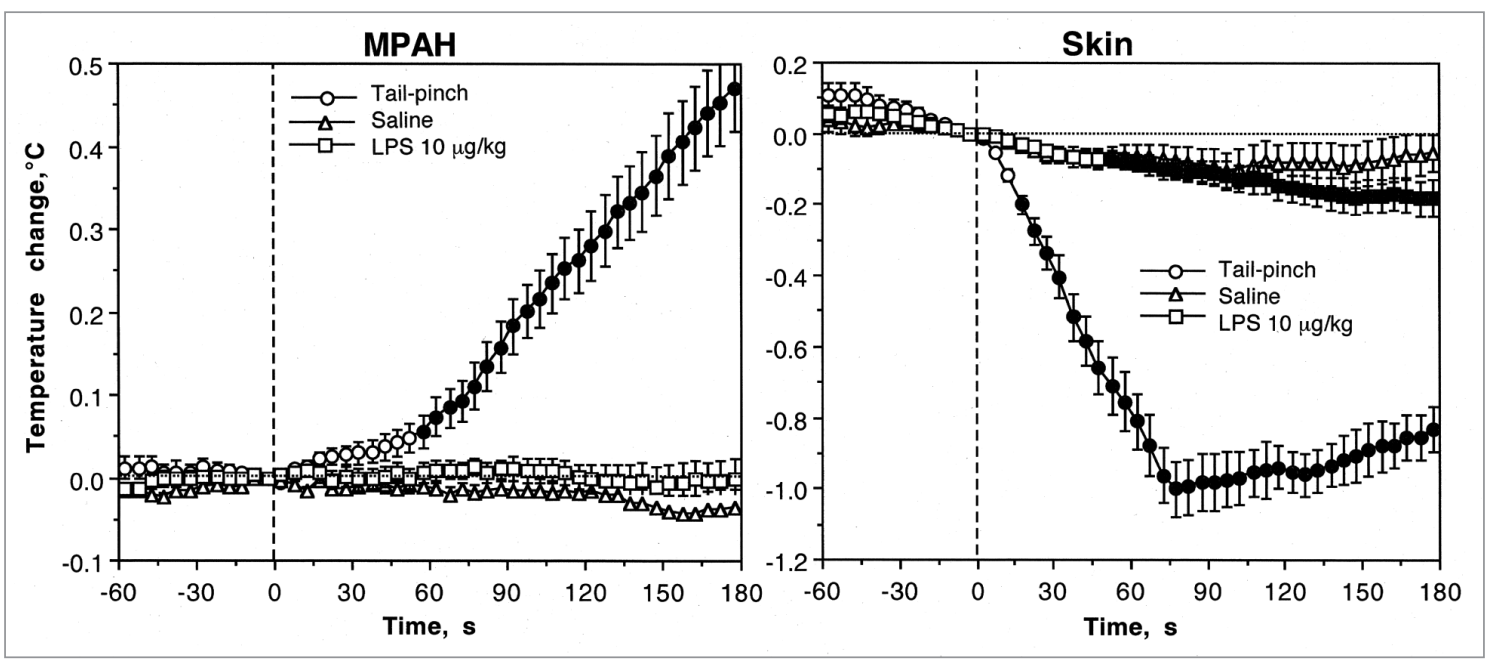

Figure 5. High-resolution evaluation of brain and skin temperature changes associated with tail-pinch and iv injections of LPS (10 $\mu \mathrm{g} / \mathrm{kg})$ and saline. Values represent relative temperature changes ( \pm standard errors) for consecutive 5 -s intervals preceding (60 s) and following (180 s) the onset of tailpinch or the injection $(0 \mathrm{~s})$. The effect of time for MPAH temperature change evaluated with one-way ANOVA with repeated measures was significant and strong for tail-pinch $\left(F_{15,495}=43.31, p<0.0001\right)$ and absent for both injections $\left(F_{15,495}=0.83\right.$ and $=0.53$ for saline and LPS, respectively). The effect of time for skin temperature change was significant and strong for tail-pinch $\left(F_{15.495}=51.69, p<0.0001\right)$, much weaker for LPS $\left(F_{15.495}=6.17, p<0.001\right)$ and absent for saline $\left(F_{15,495}=1.22, p=0.20\right)$. Individual values significantly different from baseline are shown by filled symbols. There were no significant differences in skin temperature between saline and LPS groups.

temperature influence of incoming arterial blood, showed quite different dynamics with each LPS dose. Surprisingly, with a threshold dose of LPS, this parameter transiently but significantly increased (i.e., temperature increase in the skin was stronger than in muscle) at 30-80 min post-injection, suggesting weak skin vasodilatation. Therefore, a transient vasodilatation in facial skin could be viewed as an adaptive mechanism that is invoked by excessive peripheral heat production and aimed at increased heat loss to maintain stability of body temperature. Vasodilative effects of LPS were shown on a number of peripheral vessels. ${ }^{40,41}$ This effect appears to be mediated via cytokines-, NO- and bradykinin-dependent mechanisms.

However, vascular response differed with LPS at $10 \mu \mathrm{g} / \mathrm{kg}$ dose. In this case, skin-muscle differentials clearly decreased at $-20-30$ min post-injection, and the decrease was evident for the entire $4 \mathrm{~h}$ recording interval. Therefore, the first phase of LPS-induced brain and body temperature elevation in this case coincides with vasoconstriction, which is tonically maintained for many hours after drug administration. Therefore, vasoconstriction does occur following LPS impact and is obviously contributes to overall brain and body temperature elevation. Although rapid vasoconstriction that is induced by somato-sensory stimuli is definitely centrally mediated, based on our data, it is difficult to speculate whether skin vasoconstrictive effect of LPS is central or peripheral.

Our finding of increased facial skin temperatures following iv LPS treatment at low doses contradicts data that revealed strong decreases in tail skin temperature following LPS administration at the same dose range. ${ }^{42}$ Since tail skin is the primary area of heat dissipation in the rat ${ }^{43}$ it is possible that weak warming of facial skin surfaces coexists with tail skin cooling, thus determining a total decrease in whole-body heat loss reported with systemic use of LPS $(50 \mu \mathrm{g} / \mathrm{kg}$, reviewed in ref. 13; note that these data were obtained for larger drug doses). While opposite temperature dynamics in facial and tail skin may suggest regional differences in vascular response to LPS, measurements of tail temperature in Dr. Romanovsky's study were made in partially restrained rats, with a sensor taped to the skin, and at the ambient temperatures slightly above thermoneutrality $\left(29-31^{\circ} \mathrm{C}\right)$, when tail skin vessels are maximally dilated and basal tail skin temperatures are high and close to those in body core. In contrast, basal tail skin and body core temperatures evaluated telemetrically in freely moving rats at normal ambient temperatures were much lower $\left(26-29^{\circ} \mathrm{C}\right.$ and $-37^{\circ} \mathrm{C}$, respectively) than in Romanovsky's study $\left(35.6^{\circ} \mathrm{C}\right.$ and $\left.38.4^{\circ} \mathrm{C}\right) .{ }^{44}$ Under these conditions, LPS at higher dose $(50$ $\mu \mathrm{g} / \mathrm{kg}$ ) increased body core temperature by only $1.5^{\circ} \mathrm{C}$, despite a much weaker decrease in tail skin temperatures $\left(-1^{\circ} \mathrm{C}\right)$ that was evident only with respect to saline control. To clarify the reasons for this inconsistency, it would be of interest to monitor facial and tail skin temperatures simultaneously in freely moving rats with chronically implanted sensors and examine their changes induced by somato-sensory stimuli and LPS at both normal laboratory and thermoneutral conditions. While our preliminary data using this approach suggest a similar direction of changes in facial and tail skin temperatures following salient environmental challenges, their basal values and response magnitudes drastically differ and were strongly dependent upon ambient temperatures.

\section{Experimental Procedures}

Subjects. Long-Evans male rats (Taconic, Germantown, NY), weighing 390-450 g and housed under a $12 \mathrm{~h}$ light cycle (lights on at 0700), with ad libitum food and water, were used. Protocols were performed in compliance with the Guide for the Care and Use of Laboratory Animals (NIH, Publications 
865-23) and were approved by the Animal Care and Use Committee, NIDA-IRP.

Surgery. All animals were implanted with three thermocouple electrodes as previously described. ${ }^{22}$ Animals were anesthetized intraperitoneally with $3.3 \mathrm{ml} / \mathrm{kg}$ of Equithesin (active ingredients: sodium pentobarbital, $32.5 \mathrm{mg} / \mathrm{kg}$ and chloral hydrate, 145 $\mathrm{mg} / \mathrm{kg}$ ) and mounted in a stereotaxic apparatus. Four holes were drilled through the skull: three for securing screws and one for thermocouple insertion over the medial preoptic anterior hypothalamus (MPAH, $1.3 \mathrm{~mm}$ posterior to bregma, $1.8 \mathrm{~mm}$ lateral to bregma, with $10^{\circ}$ insertion angle) using the coordinates of Paxinos and Watson (1998). ${ }^{45}$ The dura mater was retracted and the thermocouple probe was slowly lowered to the desired target depth $(8.1 \mathrm{~mm}$, measured from the skull surface). A second thermocouple probe was implanted subcutaneously along the nasal ridge with the tip approximately $15 \mathrm{~mm}$ anterior to bregma. A third thermocouple probe was implanted in the deep temporal muscle (Musculus temporalis). The probes were secured with dental cement to three stainless steel screws threaded into the skull. During the same surgery session, animals were also implanted with a jugular iv catheter. For jugular catheter implantation, a 10 $\mathrm{mm}$ incision was made in the neck to expose the jugular vein. A catheter was then inserted into and secured to the vein. The catheter was run subcutaneously to the head mount and secured with dental cement. Rats were allowed for three days' recovery and one more day of habituation to the testing environment prior the start of testing. To minimize the arousing effects of the recording environment, all rats were habituated to the chamber (placement for 6 hours) for three consecutive days before surgeries.

Experimental protocol. All tests occurred inside a Plexiglas chamber $(32 \times 32 \times 32 \mathrm{~cm})$ equipped with four infrared motion detectors (Med Associates, Burlington, VT USA) and placed inside of a sound attenuation chamber with weak $(15 \mathrm{~W})$ white illumination. Rats were brought to the testing chamber at $\sim 09: 30$ $\mathrm{AM}$ and attached via a flexible cord and electrical commutator to thermal recording hardware (Thermes 16, Physitemp, Clifton, NJ USA). A catheter extension was also attached to the exit of internal catheter, thereby allowing remote, unsignalled iv injections. Temperatures were recorded with a time resolution of $5 \mathrm{~s}$ and movement was recorded as the number of infrared beam breaks per $1 \mathrm{~min}$. Room temperature was maintained within $27-29^{\circ} \mathrm{C}$, corresponding to thermoneutral conditions ${ }^{19}$ and controlled by another thermocouple probe located in the recording chamber.

Each rat underwent at least six testing sessions with iv injections. In three sessions, rats received $0.3 \mathrm{ml}$ of LPS solution (one at $1.0 \mu \mathrm{g} / \mathrm{kg}$ and two at $10 \mu \mathrm{g} / \mathrm{kg}$ ) and in the other three- 0.3 $\mathrm{ml}$ saline. The injections were spaced so that the first, third and fifth day recorded the effects of saline, the second day recorded the effects of LPS at $1.0 \mu \mathrm{g} / \mathrm{kg}$, while the fourth and seventh days recorded the effects of LPS at $10 \mu \mathrm{g} / \mathrm{kg}$. LPS was purchased from Sigma-Aldrich (lipopolysaccharides from Escherichia coli 055:B5; L2880) and suspended in saline. All LPS solutions for each rat were prepared on the second recording day. This allowed the 1 $\mu \mathrm{g} / \mathrm{kg}$ LPS solution to be prepared fresh, whereas the $10 \mu \mathrm{g} / \mathrm{kg}$ LPS solutions were prepared, stored at $-20^{\circ} \mathrm{C}$, and later thawed out before immediate use. All injections released a $0.3 \mathrm{ml}$ volume over $20 \mathrm{~s}$. To establish thermal baselines, the rats habituated to the testing chamber for approximately 2 hours prior to all injections. Catheters were flushed with saline at the end of each recording session. During the saline sessions, the rats were exposed to a tail-pinch procedure by placing a wooden clothes pin at the base of the rat's tail for one minute.

Histology and data analysis. When recording was completed, all rats were anesthetized and decapitated, and had their brains removed for sectioning and confirmation of probe placement. Brains were cut on a cryostat into $50 \mu \mathrm{m}$ slices and placed on glass slides. All probes were located within the medial preoptic-anterior hypothalamus, as described in Paxinos and Watson (1998). ${ }^{45}$

Temperature and movement data were analyzed with different time intervals and presented as both absolute and relative changes with respect to the moment of stimulus presentation or LPS/saline administration. Based on relative temperature changes, we also calculated MPAH-muscle and skin-muscle temperature differentials that show the relationships between temperature fluctuations in these pairs of recording locations. While stronger temperature increases in brain vs. muscle are indicative of central triggering of brain temperature response, equal changes or a more rapid increase in muscle vs. brain temperature are indicative of a peripheral source of heat production, suggesting increased temperature of arterial blood inflow as the primary cause of brain hyperthermia. While a decrease or increase in skin temperature indicates increased or decreased heat dissipation to the external environment, skin-muscle differentials provide a more accurate measure of vasoconstriction/vasodilatation by excluding a slower temperature influence from arterial blood.

One-way ANOVA with repeated measures, followed by posthoc Fisher tests, was used for statistical evaluation of temperature and movement responses. Student's t-test was used for comparisons of between-site and between-condition (drug vs. saline) differences in temperature and locomotion. Between-treatment differences were evaluated based on statistical comparisons of basal brain temperatures, absolute and relative temperature changes and mean values of locomotor responses. The use of the words "increase" or "decrease" as well as "significant" refers to the presence of a statistically significant change in the parameter or in the differences between the compared groups or conditions (with at least $\mathrm{p}<0.05$ ) revealed by either ANOVA or Student's t-test. For text clarity, most of the quantitative results of statistical data evaluations are shown in Figure captions. While temperature data were recorded with high temporal resolution (5-s bins) and high-resolution analysis was used for evaluating rapid temperature fluctuations, for most analyses, data were averaged for 1 or $3 \mathrm{~min}$ to represent temperature dynamics for prolonged time intervals.

\section{Conclusions}

The present study demonstrates drastic differences in brain temperature response depending upon its triggering factors and underlying mechanisms. When this response is triggered via activation of somato-sensory pathways, i.e., via a clear neural 
mechanism, it is rapid, transient, monophasic and coupled with a strong peripheral vasoconstriction. In contrast, LPS-induced brain temperature elevation develops with definite latencies, has several phases, and is long-term at both low doses, pointing at multiple contributing factors and different underlying mechanisms. While our data suggest enhanced peripheral heat production as the primary factor responsible for brain temperature elevation by LPS at threshold doses, at higher doses this factor is supplemented by intra-brain heat production and peripheral vasoconstriction. By enhancing heat production and diminishing heat loss, these factors could determine greater and more prolonged temperature elevations with increased pyrogen doses.

\section{Acknowledgements}

This research was supported by the Intramural Research Program of the NIH, NIDA. We thank Drs. Andrey A. Romanovsky and Clark M. Blatteis for helpful comments on the issues examined and discussed in this report.

\section{References}

1. Delgado JMR, Hanai T. Intracerebral temperatures in free-moving cats. Am J Physiol 1966; 211:755-69.

2. Hayward JN, Baker MA. Role of cerebral arterial blood in the regulation of brain temperature in the monkey. Am J Physiol 1968; 215:389-403.

3. Kiyatkin EA, Brown PL, Wise RA. Brain temperature fluctuation: a reflection of functional neural activation. Eur J Neurosci 2002; 16:164-8.

4. Kiyatkin EA, Mitchum RD. Fluctuations in brain temperature during sexual interaction in male rats: An approach for evaluating neural activity underlying motivated behavior. Neuroscience 2003; 119:1169-83.

5. Smirnov MS, Kiyatkin EA. Phasic and tonic fluctuations in brain, muscle and skin temperatures during motivated drinking behavior in rats: Physiological correlates of motivation and reward. Brain Res 2010; 1310:87-102.

6. Blatteis CM. The onset of fever: new insights into its mechanism. Prog Brain Res 2007; 162:3-14.

7. Saper CB. Neurobiological basis of fever. Ann NY Acad Sci 1998; 856:90-4.

8. Romanovsky AA, Kulchitsky VA, Simons CT. "Biphasic" fever often consists of more than two phases. Am J Physiol 1998; 275:323-31.

9. Romanovsky AA, Simons CT, Kulchitsky VA, Sugimoto N. Methodology of fever research: why are polyphasic fevers often thought to be biphasic? Am J Physiol 1998; 275:332-8.

10. Dinarello CA. Infection, fever and exogenous and endogenous pyrogens: some concepts have changed. J Endotoxin Res 2004; 10:201-22.

11. Singh AK, Yiang Y. How does peripheral lipopolysaccharide induce gene expression in the brain of rats? Toxicology 2004; 201:197-207.

12. Banks WA, Robinson SM. Minimal penetration of lipopolysaccharide across the murine blood-brain barrier. Brain Behav Immunity 2010; 24:102-9.

13. Buchanan JB, Peloso E, Satinoff E. Thermoregulatory and metabolic changes during fever in young and old rats. Am J Physiol 2003; 285:1165-9.

14. Jennings G, Elia M. Effect of E. coli endotoxin on temperature, oxygen consumption and brown adipose tissue thermogenesis in rats and mice. Biosci Rep 1987; 7:517-23

15. Steiger M, Senn M, Altreuther G, Werling D, Sutter F, Kreuzer M, Langhans W. Effect of a prolonged lowdose lipopolysacharide infusion on food intake and metabolism in heifers. J Anim Sci 1999; 77:2523-32.

16. Bagby GJ, Lang CH, Skrepnik N, Golightly G, Spitzer $\mathrm{JJ}$. Regulation of glucose metabolism after endotoxin and during infection is largely independent of endogenous tumor necrosis factor. Circ Shock 1993; 39:211-9.
17. Faggioni R, Shigenaga J, Moser A, Feingold KR, Grunfeld C. Induction of UCP2 gene expression by LPS: a potential mechanism for increased thermogenesis during infection. Biochem Biophys Res Commun 1998; 6:75-8.

18. Herrington PL. The heat regulation of small laboratory animals at various environmental temperatures. Am J Physiol 1940; 129:123-39.

19. Romanovsky AA, Ivanov AI, Shimansky YP. Ambient temperature for experiments in rats: a new method for determining the zone of thermal neutrality. J Appl Physiol 2002; 92:2667-79.

20. Kiyatkin EA. Brain hyperthermia as physiological and pathological phenomena. Brain Res Rev 2005; 50:27-56.

21. Kiyatkin EA. Brain temperature homeostasis: Physiological fluctuations and pathological shifts. Front Biosci 2010; 15:73-92

22. Kiyatkin EA, Brown PL. Brain and body temperature homeostasis during sodium pentobarbital anesthesia with and without body warming in rats. Physiol Behav 2005; 84:563-70.

23. Buchanan JB, Peloso E, Satinoff E. Influence of ambient temperature on peripherally induced interleukin$1 \beta$ fever in young and old rats. Physiol Behav 2006; 88:453-8.

24. Fleshner M, Goehler LE, Schwartz BA, McGorry M, Martin D, Maier SF, Watkins LR. Thermogenic and corticosterone responses to intravenous cytokines (IL-1 $\beta$ and TNF $\alpha$ ) are attenuated by subdiaphragmal vagotomy. J Neuroimmunol 1998; 86:134-41.

25. Ootsuka Y, Blessing WW, Steiner AA, Romanovsky AA. Fever response to intravenous prostoglandin E2 is mediated by the brain but does not require afferent vagal signaling. Am J Physiol 2008; 294:1294-303.

26. Zhu M, Ackerman JH, Yablonskiy DA. Body and brain temperature coupling: the critical role of cerebral blood flow. J Comp Physiol 2009; 179:701-10

27. Feitelberg S, LamplH. Warmetonung der Grosshirnrinde bei Erregung und Ruhe. Functionshemmung. Arch Exp Path Pharmak 1935; 177:726-36.

28. McElligott JC, Melzack R. Localized thermal changes evoked in the brain by visual and auditory stimulation. Exp Neurol 1967; 17:293-312.

29. Nybo L, Scher NH, Nielson B. Inadequate heat release from the human brain during prolonged exercise with hyperthermia. J Physiol 2002; 545:697-704.

30. Kiyatkin EA. Brain temperature fluctuations during physiological and pathological conditions. Eur J Appl Physiol 2007; 101:3-17.

31. Steiner AA, Ivanov AI, Serrats J, Hosokawa H, Phayre AN, Robbins JR, et al. Cellular and molecular bases of the initiation of fever. PLoS Biology 2006; 4:1517-24.
32. Romanovsky AA, Steiner AA, Matsumura K. Cells that trigger fever. Cell Cycle 2006; 5:2195-7.

33. Szekely M, Zelenyi Z, Sumegi I. Brown adipose tissue as a source of heat during pyrogen-induced fever. Acta Physiol Acad Sci Hung 1973; 43:85-8.

34. Cannon B, Houstek J, Nedergaard J. Brown adipose tissue. More than effector of thermogenesis. Ann NY Acad Sci 1998; 856:171-87.

35. Roth J, de Souza GEP. Fever induction pathways: evidence from responses to systemic or local cytokine formation. Brasil J Med Biol Res 2001; 34:301-14.

36. Elmquist JK, Scammel E, Jacobson CD, Saper CB. Distribution of Fos-like immunoreactivity in the rat brain following intravenous lipopolysaccharide administration. J Comp Neurol 1996; 371:85-103.

37. Zhang YH, Elmquist JK, Saper CR. Lipopolysaccharide activates specific populations of hypothalamic and brainstem neurons that project to the spinal cord. Neurosci 2000; 20:6578-86

38. Elmquist JK, Saper CB. Activation of neurons projecting to the paraventricular hypothalamic nucleus by intravenous lipopolysaccharide. J Comp Neurol 1996; 374:315-31.

39. Gourine AV, Dale N, Laudet R, Poputnikov DM, Spyer $\mathrm{KM}$, Gourine VN. Release of ATP in the central nervous system during systemic inflammation: real-time measurement in the hypothalamus of conscious rabbits. J Physiol 2007; 585:305-16.

40. Cannon TR, Mann GE, Baydoun AR. Mechanisms of acute vasodilator response to bacterial lipopolysacharide in the rat coronary microcirculation. $\mathrm{Br}$ J Pharmacol 1998; 123:637-44.

41. Farias NC, Borelli-Montigny GL, Fauaz G, Feres T, Borges AC, Paiva TB. Different mechanisms of LPS-induced vasodilatation in resistance and conductance arteries from SHR and normotensive rats. $\mathrm{Br} \mathrm{J}$ Pharmacol 2002; 137:213-20.

42. Romanovsky AA, Kulchitsky VA, Akulich NV, Koulchitsky SV, Simons CT, Sessler DI, et al. First and second phases of biphasic fever: two sequential stages of the sickness syndrome? Amer J Physiol 1996; 271:244-53.

43. Gordon CJ. Thermal biology of the laboratory rat Physiol Behav 1990; 47:963-91.

44. Gordon CJ, Puckett E, Padnos B. Rat tail skin temperature monitored noninvasively by radiotelemetry: characterization by examination of vasomotor responses to thermomodulatory agents. J Pharmacol Toxicol Meth 2002; 47:107-14

45. Paxinos J, Watson C. The rat brain in stereotaxic coordinates. Sydney, Academic Press 1998. 


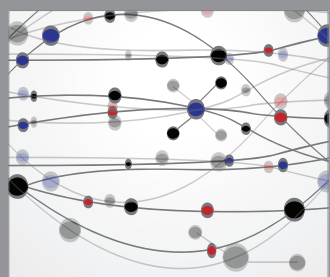

The Scientific World Journal
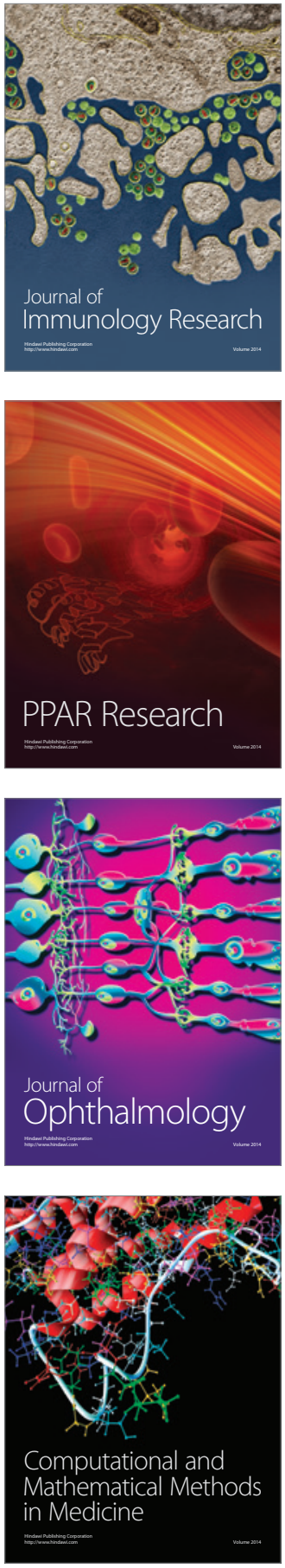

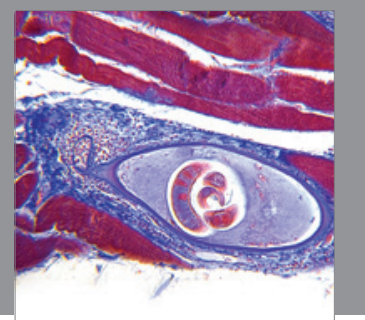

Gastroenterology

Research and Practice
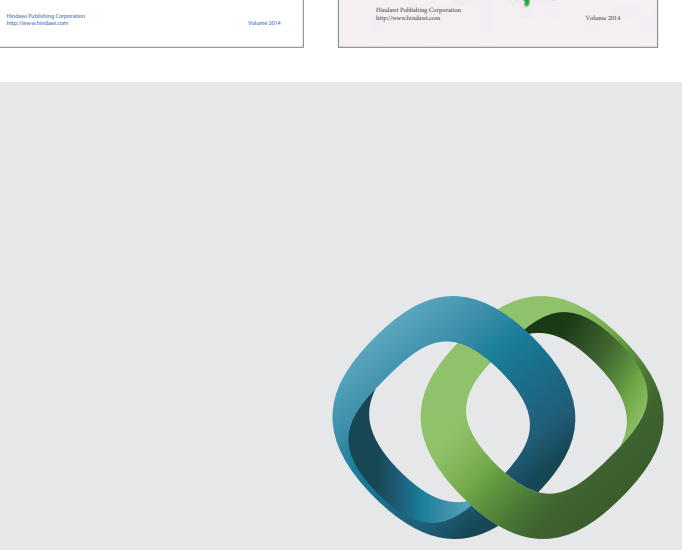

\section{Hindawi}

Submit your manuscripts at

http://www.hindawi.com
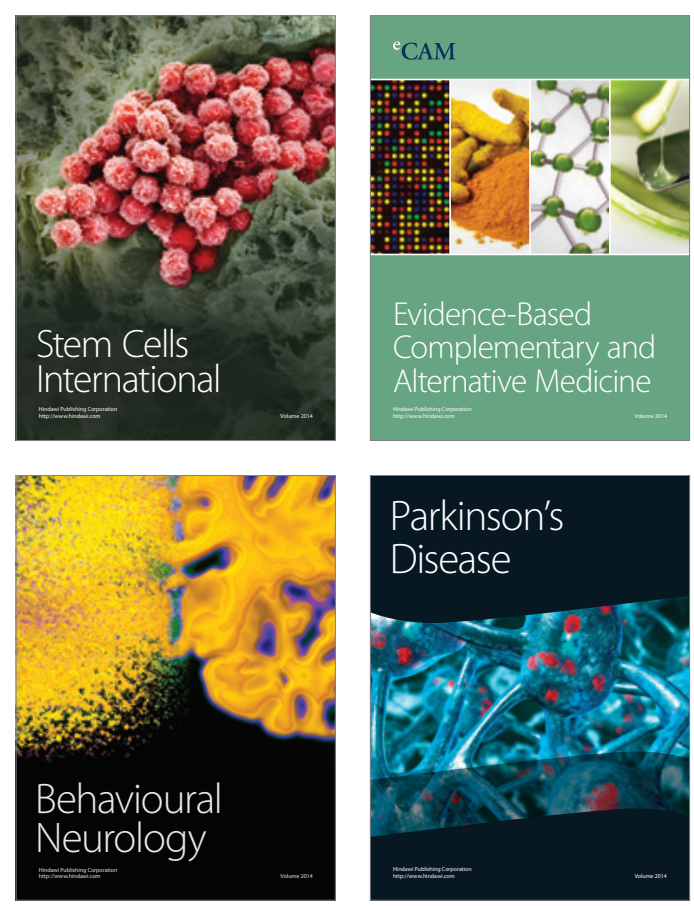

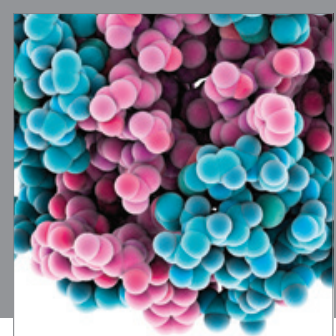

Journal of
Diabetes Research

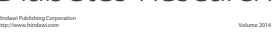

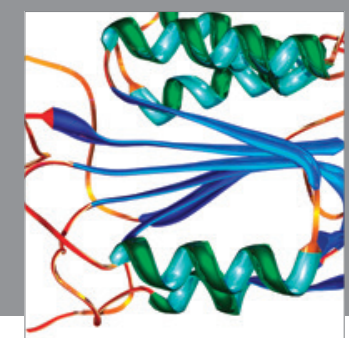

Disease Markers
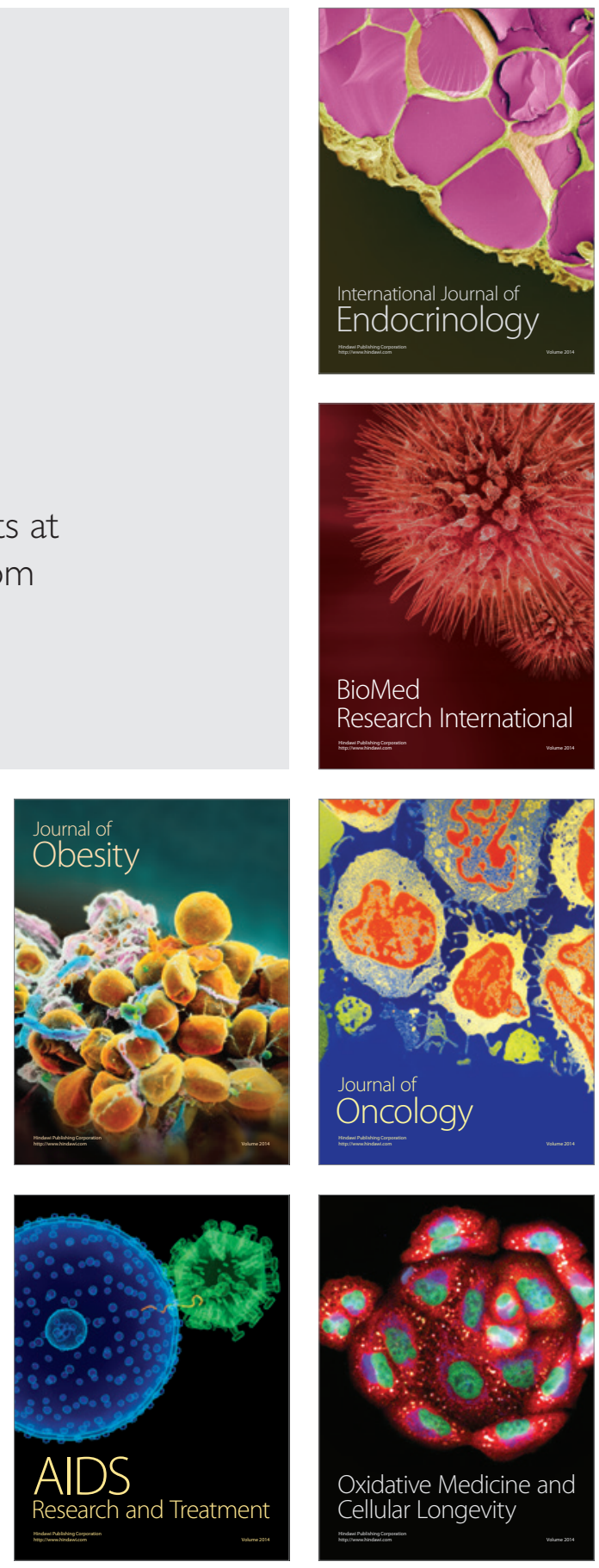